ECCOMAS

Proceedia
COMPDYN 2021

$8^{\text {th }}$ ECCOMAS Thematic Conference on Computational Methods in Structural Dynamics and Earthquake Engineering M. Papadrakakis, M. Fragiadakis (eds.) Streamed from Athens, Greece, 28 - 30 June 2021

\title{
FUNCTIONAL ACCEPTANCE TESTS FOR THE SEISMIC ISOLATION DEVICES OF THE NEW SAN GIORGIO BRIDGE IN GENOA
}

\author{
Marcello Cademartori ${ }^{1}$, Andrea Miano ${ }^{2}$, Antimo Fiorillo ${ }^{2}$, Alessandro Aliotta ${ }^{1}$, Marco \\ Di Ludovico ${ }^{2}$, Andrea Prota ${ }^{2}$, Simone Dellacasagrande ${ }^{1}$, Alaeddine Fatnassi ${ }^{1}$, Daniele \\ Pastorelli $^{1}$ \\ ${ }^{1}$ RINA Consulting \\ Via Cecchi 6, Genoa, Italy \\ \{marcello.cademartori,alessandro.aliotta,s.dellacasagrande,alaeddine.fatnassi,daniele.pastorelli\}@rina.org \\ ${ }^{2}$ Department of Structures for Engineering and Architecture, University of Naples Federico II \\ Via Claudio 21, Naples, Italy \\ \{andrea.miano,a.fiorillo,diludovi,a.prota\}@unina.it
}

\begin{abstract}
The New San Giorgio Bridge has been built in a very short time between April 2019 and August 2020 in order to reconnect the highway system of the western part of Italy after the tragedy of the collapse of the Morandi Bridge, built in 1967 by prof. Riccardo Morandi. The new viaduct has a steel concrete composite closed section for the deck, a structural scheme of continuous beam with a total of 19 spans supported by elliptical concrete box piers with a maximum height of $40 \mathrm{~m}$. The bridge is seismically isolated by means of friction pendulum devices and multidirectional bearings. In the transverse direction the seismic devices, except for two piers, are a combination of friction pendulum and shear fuses. The paper describes the construction supervision process for the functional acceptance tests of the bearing devices with respect to the Italian National Law, NTC2018, European norms EN15129 and EN1337, and in fulfilment with the Technical Specifications issued by the Italian Railway system (RFI) and Italian State Road Association (ANAS). The paper highlights the main issues encountered during the application of the several specifications for the certification and acceptance processes. A description of the different functional tests required during the construction supervision process is presented as well as an overview of the different acceptance criteria of the tests results. The aim is to provide useful recommendations for technicians involved in the definition of the seismic acceptance tests for bearing devices for highway bridges in the Italian and European contest.
\end{abstract}

Keywords: New San Giorgio Bridge, Seismic isolation, Curved surface sliders, Certification, Acceptance Tests, Benchmark test. 


\section{INTRODUCTION}

On the $14^{\text {th }}$ August 2018 the highway Viaduct of the A10, one of the strategic roads of the northern part of Italy, collapsed. This tragedy caused the death of 43 people that were crossing the bridge in that moment.

The viaduct was designed between the 1964 and 1967 by the famous Italian Engineer prof. Riccardo Morandi and it was a cable stayed bridge structure with reinforced concrete post tensioned cables.

On December 2018, RINA Consulting was charged to develop the Project and Management, the Construction Supervision, the Health and Safety Management for the demolition of the Morandi Bridge and for the reconstruction of the Polcevera Viaduct, the Genoa new San Giorgio Bridge (called for brevity San Giorgio Bridge in the following) designed by the Arch. Renzo Piano. In this process, RINA took the role of technical and administrative support of the Commissioner all along the design process and it has been present during all the activities of demolition and reconstruction from the beginning to the end.

The San Giorgio Bridge has been designed by Italferr, starting from the concept of Arch. Renzo Piano, and has been built by a consortium called PerGenova and composed by WeBuild and Fincantieri. Because of the emergency, the reconstruction of the bridge was a very challenging task as it involved in parallel the project development and the construction phase.

As part of the Construction Supervision role, an important activity is the quality assurance that requires the control of the construction products on site as well as in the factories.

One of the most important task related to the quality control for the San Giorgio Bridge was related to the qualification and acceptance tests of the seismic devices and bearings installed in the viaduct to isolate the steel-concrete deck from the piers. This require the development of a detailed "Testing Protocol" as part of the quality control activity and this document has been deployed taking into account: i) design specifications according to the design developed by Italferr; ii) drawings developed by the manufacturer Maurer SE and FIPMEC; iii) European norms and Italian specifications; iv) technical capabilities of the testing devices present worldwide.

Figure 1 shows the San Giorgio Bridge after the construction phase. The testing program according to the construction and project plan aiming at match the main milestones in terms of deck launching and the time required to perform the tests. To this aim, the required functional tests have been organized in compliance with the construction plan requirement with the support and contribution of all the laboratories charged to execute the tests. Differently from other general design projects, in this case the acceptance process corresponds with the certification process, generating many issues normally not present. Indeed, in this case, the devices are not commercially produced elements, but have been realized "ad hoc" for the bridge, due to the requirements from the design specifications (e.g., the presence of the 100 meter spans and the condition of only 2 devices on each pier led to significant dimensions and acting forces for the devices).

This paper presents a detailed description of the whole testing program with a particular focus on the acceptance tests protocol, as well as a summary of the performance of the devices during the specified static and dynamic tests. Despite the demanding testing requirements in the new European norm, the results proved that the proposed design of the isolators successfully fulfilled the performance required for the acceptance of the devices. 


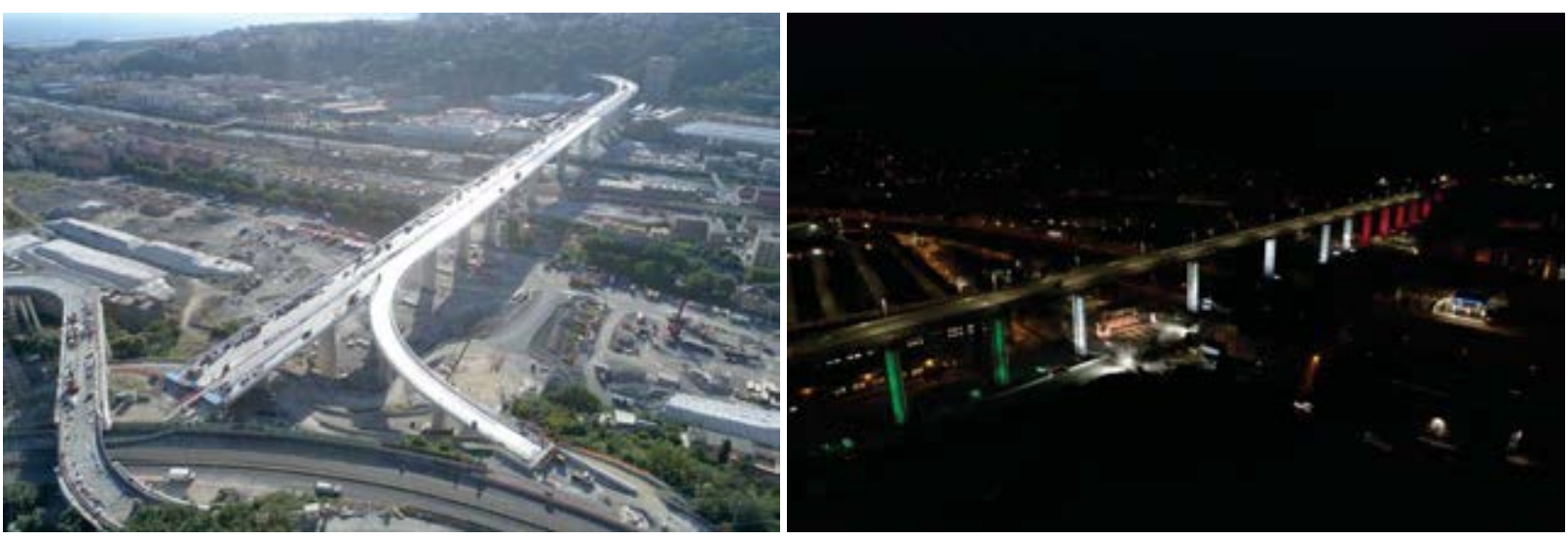

Figure 1: Pictures of the San Giorgio Bridge.

\section{THE SEISMIC ISOLATION SYSTEM OF THE SAN GIORGIO BRIDGE}

The San Giorgio Bridge has a length of $1067 \mathrm{~m}$ and the road level is at a height of $40 \mathrm{~m}$. The bridge has a steel-concrete composite deck with an overall width of $29.8 \mathrm{~m}$ and with a distance between the supports of $7 \mathrm{~m}$. From the structural point of view, the bridge is a continuous beam with all spans' length of $50 \mathrm{~m}$ except for the three spans in the center (with a length of $100 \mathrm{~m}$ and for the spans near the abutments that are shorter).

The deck is seismically isolated from the piers. The supporting scheme has been designed to withstand the seismic actions, wind actions and thermal actions, particularly relevant in a bridge with a length of $1067 \mathrm{~m}$ without intermediate movement joints. Figure 2 shows a plan view of the bridge.

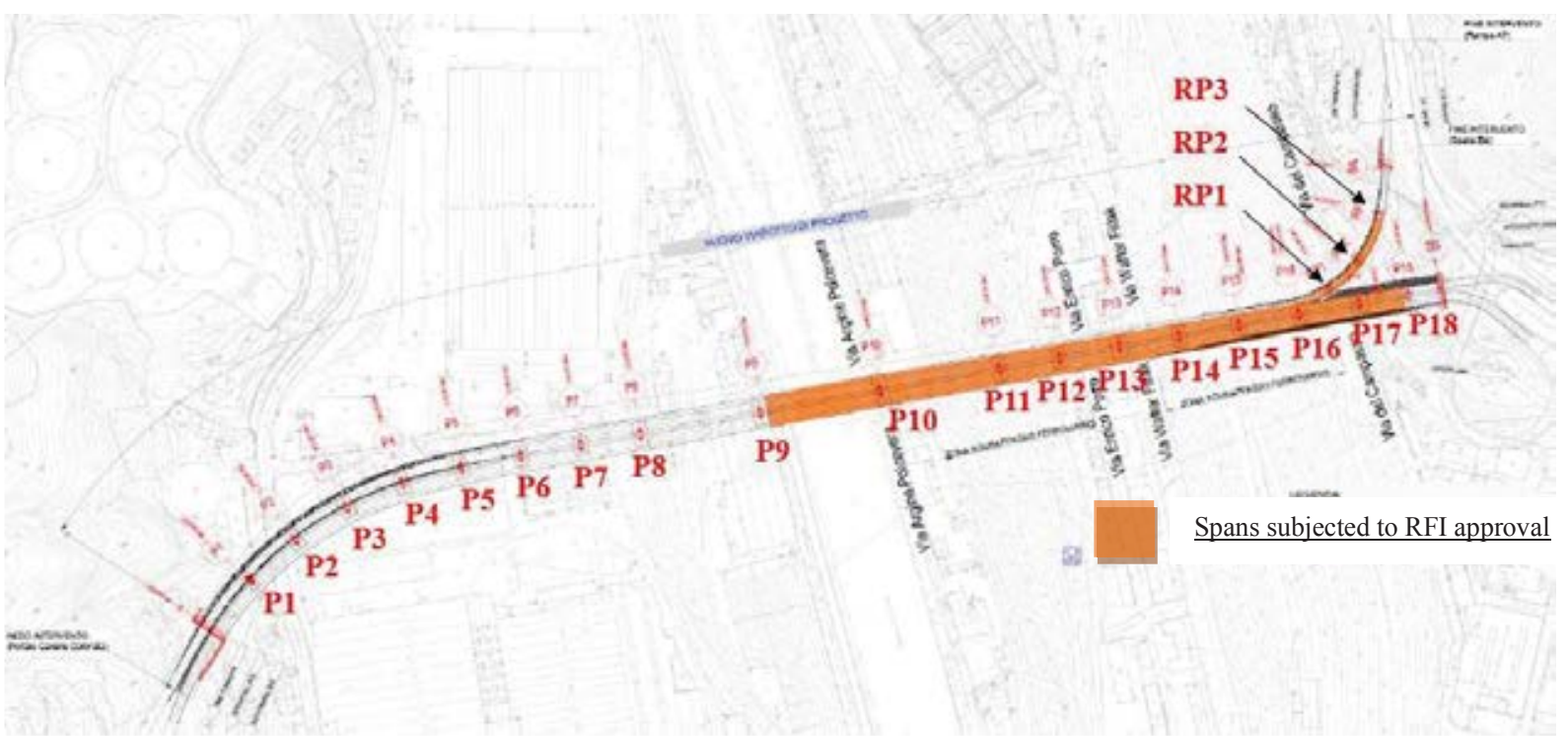

Figure 2: Plane view of the San Giorgio Bridge.

The bridge is equipped with the following devices (between the parenthesis, the nomenclature as per the UNI EN15129 [1] is reported), according to the plan view of Figure 2: i) two curved surface sliders (friction pendulum) on each pier from P2 to P17 with shear fuses for the device on the north side (combined devices: curved surface sliders + fuses) and without shear fuses for the device on the south side; ii) two multidirectional bearings on pier P1 and P18; iii) two multidirectional bearings on the abutments $\mathrm{A}$, west side, and abutments $\mathrm{B}$, east side; iv) a guided bearing in the center of the abutments; v) two curved surface sliders on the 
piers RP1-RP2-RP3 of the ramp; vi) an elastomeric device in the center of the abutment A of the ramp; vii) two multidirectional bearings on the abutment $A$ of the ramp.

The supporting system of the bridge has been designed to guarantee horizontal flexibility of the bridge during the seismic actions; this to increase the structural period of vibration and to reduce the seismic acceleration, while at the same time to limit the movement due to the wind actions. For this reason, the shear fuses are placed on the isolators in the south side of the piers from P2 to P17: these fuses aims at ensuring a fixed behaviour in the transversal direction for the serviceability limit states while they will break off in case of seismic event.

To avoid the direct contact between steel deck and concrete piers in case of extreme seismic events, the deck is also equipped with seismic restraints in the transversal direction in each pier and in the transversal and longitudinal direction in the abutments. Movement joints are provided only at the three abutments.

This paper focuses on the seismic certification and acceptance protocol followed for the curved surface sliders, friction pendulum and, then, for the categories i) and v) previously described. Moreover, a detailed discussion is provided with reference to the acceptance tests for the devices under investigation.

\subsection{Seismic behavior of the curved surface sliders and combined devices}

Curved surface sliders are seismic isolators that provide the four main functions of the base isolation (see UNI EN 15129, 3.1.26) through an appropriate arrangement of curved sliding surfaces; they use the characteristics of a pendulum to increase the natural period of the isolated structure. Several experimental and analytic studies on single and double concave surface sliders are present in literature, concerning different critical issues and the uniaxial and/or the biaxial response (e.g., [2-5]).

The curved main sliding surface of Curved Surfaces Sliders provides a restoring force at displacement $d$.

Energy is dissipated by friction due to movement in the main sliding surface. Rotations of the structure are accommodated by the secondary sliding surface.

Figure 3 shows the functional principle and main elements of curved surface sliders for the type A (see Table 1) curved surface sliders of the San Giorgio Bridge.

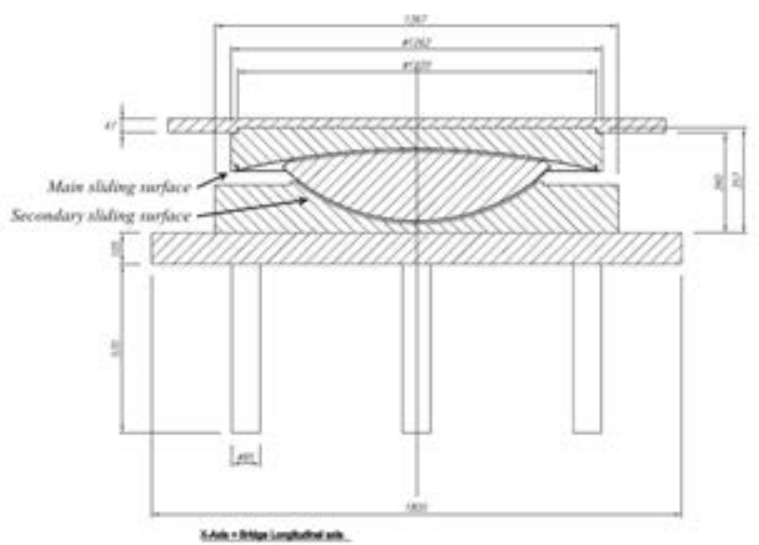

(a)

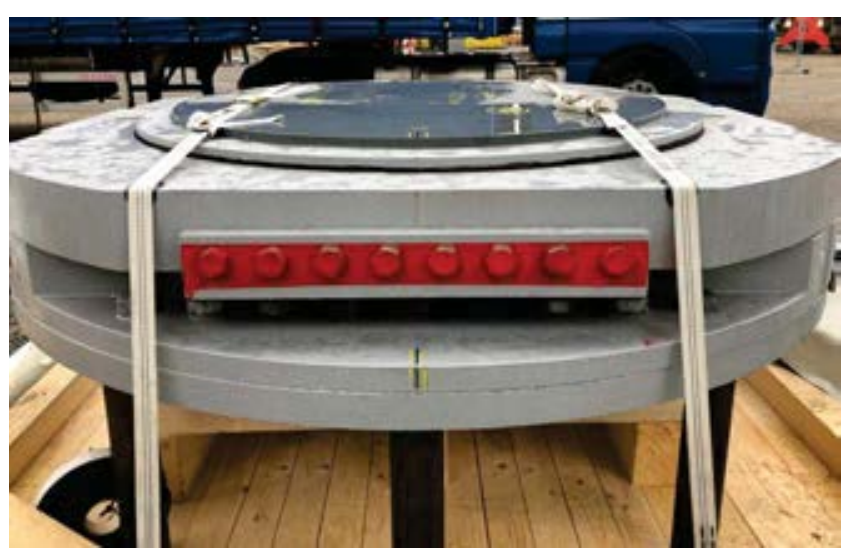

(b)

Figure 3: (a) Functional principle and main elements of Curved Surface Sliders for the type A (see Table 1) curved surface sliders of the San Giorgio Bridge; (b) picture of the device.

The constitutive law of this typology of devices in the horizontal plane, under constant axial load, is usually described with the following relationship [6]: 


$$
F=M g \cdot \mu_{d i n}+\frac{M g}{R} \cdot d
$$

where:

$\mathrm{F}=$ horizontal force

$\mathrm{Mg}=\mathrm{N}$ is the vertical force

$\mathrm{R}=$ radius of curvature of the device

$\mathrm{d}=$ horizontal displacement

$\mu_{\text {dyn }}=$ dynamic friction coefficient

The resultant hysteresis loop is reported in Figure 4.

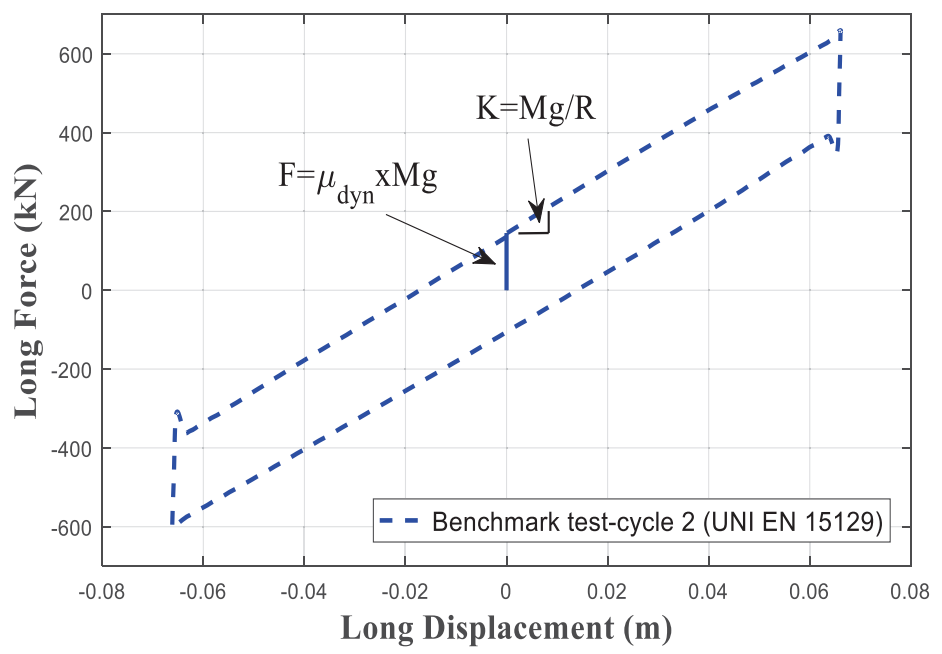

Figure 4: Force-Displacement relationship of the curved surface slider referred to the benchmark-cycle 2 test for one of the bridge devices.

Figure 5 shows the break-away friction, also called stick-slip effect, that is the forces recorded when the motion of the device starts [7], as well as the increase of force that is recorded with the inversion of the velocity.

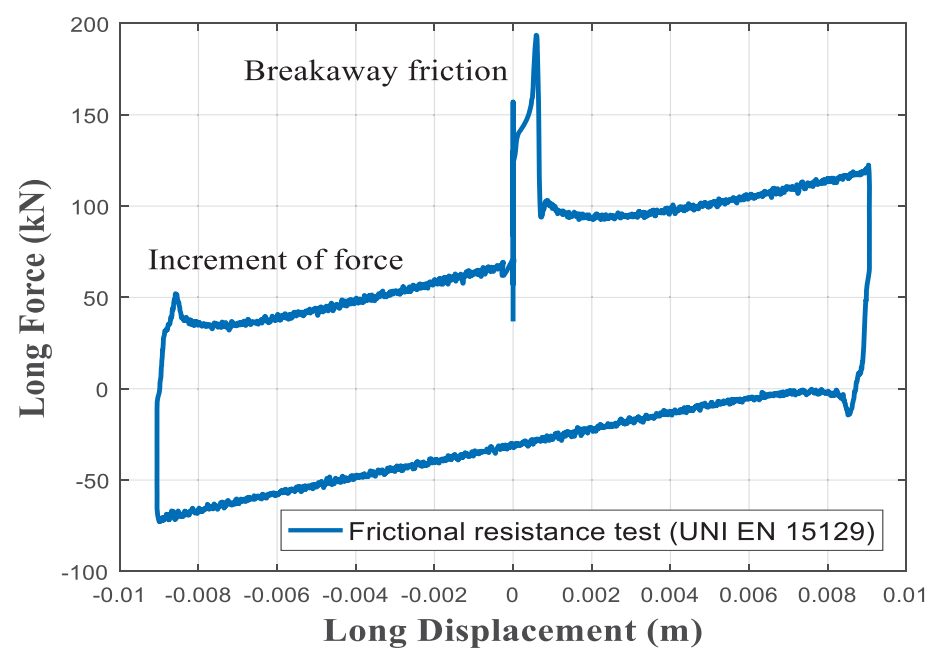

Figure 5: Experimental behavior of curved surface slider referred to the frictional resistance test for one of the bridge devices.

For the San Giorgio Bridge, the curved surface sliders are combined, in the transversal direction, with shear fuses. In this case, the device acts as a friction pendulum in the longitudi- 
nal direction while for the transverse direction is fixed until a specific value of force is reached. Once the break-away force is reached the device has the same behavior in both the horizontal directions. The main objective of the combination of the shear fuses for the viaduct is to guarantee the benefits of the base isolation and to limit horizontal displacement under transversal wind loads.

\section{THE CERTIFICATION AND ACCEPETANCE PROCESS FOR THE CURVED SURFACE SLIDERS IN THE EUROPEAN REGULATIONS FRAMEWORK}

This chapter describes the certification and acceptance process for the curved surface sliders. Clearly, the design is directly linked to the recommendations of the country of realization of the construction. In the case of the San Giorgio Bridge, the Italian national code (NTC 2018, [8]) is inserted inside the context of European codes and specifically the UNI EN 15129 [1] and the UNI EN 1337 [9]. NTC 2018 [8] clearly separate the tests related to the certification process from the tests related to the acceptance process. This separation between certification and acceptance processes and the relative definition of the tests plan for the curved surface sliders is in perfect agreement with the suggestions of the UNI EN15129 [1] and UNI EN1337 [9]. Moreover, according to NTC 2018 [8] specific tests should be added based on the different type of isolators. For the case study of the San Giorgio Bridge, the curved surface sliders, described in Section 2, did not exist at the moment of the bridge design. They have been designed from the company ad hoc for the San Giorgio Bridge. Thus, in this case the certification and acceptance processes develop in parallel.

For the certification procedure, NTC 2018 (11.9.2) [8] requires that the anti-seismic devices must comply with the European standard harmonized UNI EN 15129 [1] and have the CE marking. The system of evaluation and verification of the constancy of performance, provided for in the aforementioned UNI EN 15129 [1], is applied. The certification procedures are intended to demonstrate that the device is capable of maintaining its functionality under the conditions of use envisaged throughout the life of the project.

For the acceptance procedure, NTC 2018 (11.9.3) [8] requires mandatory on-site acceptance checks for all the types of devices; furthermore, the Works Supervisor, before in-situ implementation, verifies the required certification documentation, and refuses the nonconforming devices. The Works Supervisor should also carry out the geometric verification and dimensional tolerances. The acceptance tests can be performed and certified by a laboratory that is able to guarantee adequate competence, equipment and organization. For the curved surface sliders, the methodology for the tests acceptance plan definition and for the relative evaluation criteria are those reported in the UNI EN 15129 [1] with reference to the Factory Production Control tests. Then, it is possible to use, for the purposes of acceptance tests, also the Factory Production Control tests carried out within the certification process of the devices in accordance with UNI EN 15129 [1] if (conditions respected for the case study San Giorgio Bridge): i) the sampling of the devices was carried out, on the lots destined for the specific site, by the Works Supervisor of construction site; ii) the tests are performed and certified by a laboratory with adequate expertise, equipment and organization; iii) the aforementioned certificates explicitly indicate the construction site (s) where the supply is used.

Finally, the devices subjected to certification or acceptance tests may be used in construction only if: i) the elements stressed in the non-linear field are replaced or if their resistance to low-cycle fatigue is at least one order of magnitude higher than the number of test cycles; ii) and in any case only after that they are subject to verification of their perfect integrity and full functionality.

The number of devices subjected to acceptance tests for the curved surface sliders is provided in Section NTC 2018 11.9.8.1 [8]. The acceptance tests must involve at least $20 \%$ of the 
devices, in any case not less than 4 and no more than the number of devices to be implemented.

\subsection{UNI EN 15129}

The UNI EN 15129 [1] standard is the European standard for the description of the process of certification and acceptance for the curved surface sliders. The UNI EN 15129 [1] requirements have been integrated with additional tests required by national recommendations. However, a brief summary of the entire process of certification and acceptance processes is summarized herein. Chapter 10 of UNI EN 15129 [1] concerns the Assessment and Verification of the Constancy of the Performance (AVCP). It specifies that the conformity of the antiseismic devices to the requirements of UNI EN 15129 [1] and to the performance declared from the producer have to be demonstrated through: i) type-product identification; ii) check of production in factory from the fabricator, including the assessment of the product.

Type-product identification is represented by the type tests that form the certification process together with the acquisition of the CE marking. According to UNI EN 15129 (10.2) [1], all the mechanical features of the sliders needed for the design to guarantee the service life, should be determined through type tests on full scale sliders, also including cyclic tests. The tests results should confirm the representative values of the mechanical features of the sliders.

Check of production in factory is represented by the factory production control (FPC) tests that form the acceptance process. According to UNI EN 15129 (10.3) [1], the fabricator should keep a FPC system to ensure that the sliders are in compliance with the performance declared in their essential features. The FPC includes also some specific tests for the devices and the check on the raw materials. These general rules are valid for all type of sliders, while the curved surface sliders are specifically analysed in Section 8.3.4 of UNI EN 15129 [1], defining the type tests (8.3.4.1) and the FPC tests (8.3.4.2).

Then, coherently with the purpose of this work, a focus is herein dedicated to the FPC tests to complete the acceptance of the devices. Section 8.3.4.2 of UNI EN 15129 [1] presents the tests to be implemented for the curved surface sliders (see Figure 6). In order to complete the FPC checks, the following tests are required:

a) Vertical load bearing capacity (see Sect. 8.3.1.2.2.2 and 8.3.4.1.2 of UNI EN 15129 [1]);

b) Frictional resistance force under service conditions (see Sect. 8.3.1.2.2.5 and 8.3.4.1.3 of UNI EN 15129 [1]);

c) Test run P1, also called benchmark test (see Sect. 8.3.1.2.2.6 and 8.3.4.1.5 of UNI EN $15129[1])$.

The testing of raw materials and constituents shall be carried out in accordance with Tab. 16 of UNI EN 1337- 2:2004 [9] or, in case of other sliding materials, according regulations provided in the respective European Technical Approval (ETA).

In conclusion, Figure 6 summarizes the acceptance protocol of curved surface sliders in an European framework, based on recommendations described in this chapter. Note that in addition to the required tests from the UNI EN 15129 [1], additional tests recommended from the national codes and from other authorities involved in the project can be added to the tests protocol. Indeed, this was one of the main challenge of the acceptance test procedure: to ensure the compliance to all the European and Italian codes and at the same time to guarantee the fulfilment of the technical specifications issued by the Italian Railway system (RFI) and by the Italian State Road Association (ANAS). 


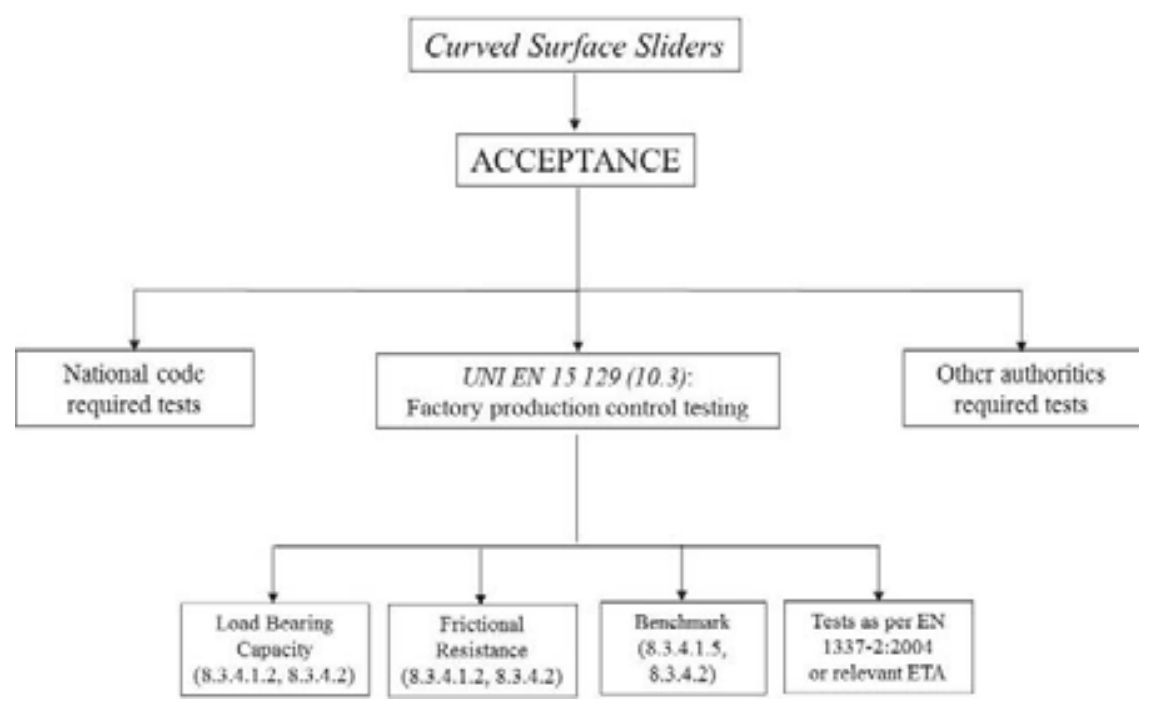

Figure 6: Flow-chart of acceptance protocol for the curved surface sliders.

In the case study of the San Giorgio Bridge, the Italian national code (NTC 2018, 11.9.8, [8]) suggests adding to the protocol another test, called Quasi Static test. Moreover, it is to note that in the case study of the San Giorgio Bridge, also the Technical Specifications issued by the Italian Railway system (RFI) and Italian State Road Association (ANAS) have been considered in the acceptance process. In particular, additional tests have been added to the acceptance protocol tests based on the recommendations of RFI, that in some cases proposed tests conceptually similar to the ones from UNI EN 1529 [1], but using different load conditions/setup conditions/acceptance parameters. In this paper, the acceptance tests required by the RFI specifications are not treated.

\section{ACCEPTANCE PROTOCOL TESTS: THE SAN GIORGIO BRIDGE CASE STUDY}

The main objectives of the testing program carried out for the San Giorgio Bridge were: i) to guarantee the fulfilment of the applicable technical norms; ii) to guarantee the respect of the design planned performances; iii) to investigate the behaviour of the devices with respect to the comments and prescriptions issued by the different authorities during the approval process of the project (e.g. Technical Authority of the Italian Minister of Transportation and comments by the Commissioning Authority); iv) to investigate the durability and long-term behaviour of the devices applying all the tests available as per the most updated state of the art in this field.

All the above objectives have been addressed aiming at the same time at the respect of the project and construction plan.

Pursuing that the codes and specifications cannot regulate every aspects of complex phenomena, a pro-active role of the Construction Supervision team, in strong cooperation with the designer with the manufacturer and with the laboratories, has been deployed with the common purpose to verify the performance of the seismic devices while at the same time respecting the construction program.

The seismic acceptance tests have been performed in different testing facilities depending on their loading and displacement capacity and at the same time to make feasible the execution of different tests in parallel. Each testing facility was used for a similar group of devices to obtain more comparable results for similar bearings. The following laboratories have been involved in the experimental program: 
- SismaLab under the supervision of the Polytechnic of Milan for the device of the ramp and for the shear fuses;

- $\quad$ Polytechnic of Milan using the external premises of FIP-MEC in Padua for the breakaway tests of the combined device friction pendulum + fuses;

- $\quad$ Eucentre Lab in Pavia for friction pendulum and multidirectional bearings.

This section presents the acceptance protocol (UNI EN 15129 [1] FPC tests plus national code required tests) for the case study of the San Giorgio Bridge. Note that additional tests on the conformity of the characteristics of the materials in accordance with Tab. 16 of UNI EN 1337- 2:2004 [9] have been implemented. Moreover, under the supervision of the RFI specialists, other tests have been carried out based on their protocol. Table 1 reports the under investigation, the type of tests performed and the standard to which each test refers (tests done in accordance with the RFI specifications are not included here).

\begin{tabular}{|c|c|c|c|c|c|}
\hline CURVED SURFACE SLIDERS TYPE & PIER & \multicolumn{4}{|c|}{ TEST } \\
\hline $\begin{array}{l}\text { TYPE A: } 3 \text { SLIDERS ON } \\
\text { THE } 100 \text { METERS BAYS }\end{array}$ & $\begin{array}{c}\mathrm{P} 9 / 3, \mathrm{P} 10 / 3 \text { and } \\
\mathrm{P} 11 / 2\end{array}$ & \multirow{3}{*}{$\begin{array}{c}\text { Load } \\
\text { Bearing } \\
\text { Capacity } \\
\text { UNI EN } \\
15129\end{array}$} & \multirow{3}{*}{$\begin{array}{c}\text { Frictional } \\
\text { Resistance } \\
\text { UNI EN } \\
15129\end{array}$} & \multirow{3}{*}{$\begin{array}{l}\text { Benchmark } \\
\text { UNI EN } \\
15129\end{array}$} & \multirow{3}{*}{$\begin{array}{l}\text { Quasi- } \\
\text { Static } \\
\text { NTC } \\
2018\end{array}$} \\
\hline $\begin{array}{l}\text { TYPE B: } 4 \text { SLIDERS ON } \\
\text { THE } 50 \text { METERS BAYS }\end{array}$ & $\begin{array}{c}\mathrm{P} 2 / 3, \mathrm{P} 13 / 3, \mathrm{P} 2 / 2 \text { and } \\
\mathrm{P} 12 / 2\end{array}$ & & & & \\
\hline $\begin{array}{l}\text { TYPE C: } 4 \text { SLIDERS ON } \\
\text { THE BRIDGE RAMP }\end{array}$ & $\begin{array}{c}\mathrm{RP} 3 / \mathrm{R} 2, \mathrm{RP} 3 / \mathrm{R} 3, \mathrm{RP} 2 / \mathrm{R} 2 \\
\text { and RP1/R2 }\end{array}$ & & & & \\
\hline
\end{tabular}

Table 1: Summary of devices under investigation and relevant tests (see Figure 2 for labels).

The following nomenclature is used: the number after "P" refers to the pier number as reported in Figure 2, while the number after the "/ refers to the location of the devices on the pier (" 3 " for the northern support and " 2 " for the southern supports). The supports located on the southern side are combined devices (curved surface sliders + shear fuses).

The sliders types have been in depth discussed in Section 2 while the present section focuses on a critical discussion on the main issues occurred during the test program:

- Because the devices were not commercially produced elements, but have been realized " $a d$ hoc" for the bridge, due to the requirements from the design specifications, the acceptance tests were carried out in parallel with those related to certification process. The UNI EN15129 [1] does not require full test for the combined devices in the acceptance tests protocol while it suggests only one test in the certification process. This may lead to significant issues in the cases in which the certification and acceptance processes correspond; indeed, high priority should be given to the combined device test, because it is crucial to verify that unexpected interferences between the components of the devices, that can affect the behavior of the whole device, are avoided. For the case study of the San Giorgio Bridge, the Construction Supervision team required the implementation of specific tests for the combined devices (tests in the transversal direction, break away tests on two sacrificial devices among others); however, they are not discussed in detail in this paper. During the combined tests, small modifications/upgrading can be necessary, such as in the case study, and this clearly point out that such a test should occur before the single components tests;

- The vertical loads are sometimes very high in particular for modern bridges (i.e. 1.3 $\mathrm{N}_{U L S}=$ axial load from gravity load combination for ultimate limit states is required for the load bearing capacity test). This could imply that testing facilities may have not adequate capacity. These constraints should be taken into account in the design of structures/infrastructures to easily allow to tests the components (potentially in 
the same geographical region of the construction), based on the current instrumentations availability of the laboratories; otherwise a significant increase of time and cost for testing not conventional devices should be considered. The norms should also provide specific procedures in such cases in order to simplify the whole procedure, by keeping the same safety level. The same problems may occur in terms of displacement requirements;

- the design of small friction values may imply that the calibration forces of the laboratory equipment can be comparable to forces to be measured; this can strongly affect the tests results.

Furthermore, as discussed in the following sections, the interpretation of some tests of UNI EN 15129 [1] may in some case not univocal or not always quantitative (e.g., see Faverage definition in the next Section).

From here, a specific focus is dedicated to the benchmark test, that is the more important dynamic test inside the requirements of UNI EN 15129 [1] in the acceptance phase.

\subsection{Benchmark test}

In the context of the curved surface sliders acceptance tests, the benchmark test is required from UNI EN 15129 [1] with two purposes:

1) Verification of cycle stability in terms of fluctuation of the test force with respect to the average force;

2) Verification of the dynamic friction coefficient.

The load application method (UNI EN 15129 8.3.4.1.5 [1]) is reported as follows in Table 2 , where $d_{b d}$ is defined as the maximum design displacement of the device. During the test, a permanent vertical load $\mathrm{N}_{\mathrm{sd}}$ (seismic combination) was applied to the device.

\begin{tabular}{|c|c|c|c|c|c|}
\hline Type of test & Test run & $\begin{array}{c}\text { Compression } \\
\text { load } \mathrm{N}_{\mathrm{s}}[\mathrm{kN}]\end{array}$ & $\begin{array}{c}\text { Displacement } \\
\mathrm{d}_{0}[\mathrm{~mm}]\end{array}$ & $\begin{array}{c}\text { Peak velocity } \\
\mathrm{v}_{0}[\mathrm{~mm} / \mathrm{s}]\end{array}$ & $\begin{array}{c}\text { Number of } \\
\text { complete cycles }\end{array}$ \\
\hline Benchmark & $\mathrm{P} 1$ & $\mathrm{~N}_{\mathrm{sd}}$ & $1 \times \mathrm{d}_{\mathrm{bd}}$ & 50 & 3 \\
\hline
\end{tabular}

Table 2: Load application method for the benchmark test (UNI EN 15129 8.3.4.1.5 [1]).

About the test acceptance criteria, according to UNI EN 15129 8.3.1.6 [1], the movement in the sliding surfaces shall be regular and uniform and without producing any type of vibrations such as those induced by the stick-slip phenomenon. The force oscillation needs to be contained inside a variation range of $+/-5 \%$ of the average restoring force, at any level of bearing displacement up to $85 \%$ of the maximum displacement required from the test protocol. The average restoring force shall be obtained from the best-fit straight line determined by the least square interpolation of the response between $+/-85 \%$ of the maximum displacement.

Then the requirement for the satisfaction of the test can be summarized as:

$$
\text { for }(-0.85) \mathrm{d}_{\mathrm{bd}}<\mathrm{d}<(+0.85) \mathrm{d}_{\mathrm{bd}}: \mathrm{F}_{\text {regression- }}\left(0.05 \mathrm{~F}_{\text {average }}\right)<\mathrm{F}_{\text {test }}<\mathrm{F}_{\text {regression }}+\left(0.05 \mathrm{~F}_{\text {average }}\right)
$$

Moreover, the dynamic friction coefficient needs to fall within the limits specified by the Structural Engineer under the testing conditions specified in UNI EN 15129 8.3.4.1.5 [1]. Then, it is considered necessary to verify that the dynamic friction coefficient deriving from the test falls within the lower and upper bounds used in the design phase. The following check can be performed by calculating the average dynamic friction using the following formulation:

$$
\mu=\frac{H}{2 \cdot\left(d^{+}+\left|d^{-}\right|\right) \cdot V_{\text {load }}}
$$


where: $\mathrm{H}=$ area subtended by the curve, $\mathrm{d}+=$ maximum displacement, $\mathrm{d}-=$ minimum displacement, $\mathrm{V}_{\text {load }}=$ vertical load. This value should vary inside the lower/upper bound range.

Then, the final requirement is that:

$$
\mu_{\text {lower bound }}<\mu<\mu_{\text {upper bound }}
$$

As for example, the results of the benchmark test on $\mathrm{P} 10 / 3$ slider (label producer MAU112E19-10) is discussed herein. Figure 7 shows the three force-displacement cycles of the test. Moreover, the displacements-time and forces-time plots for the 3 cycles are presented in Figure 8.

BENCHMARK - 3 CYCLES

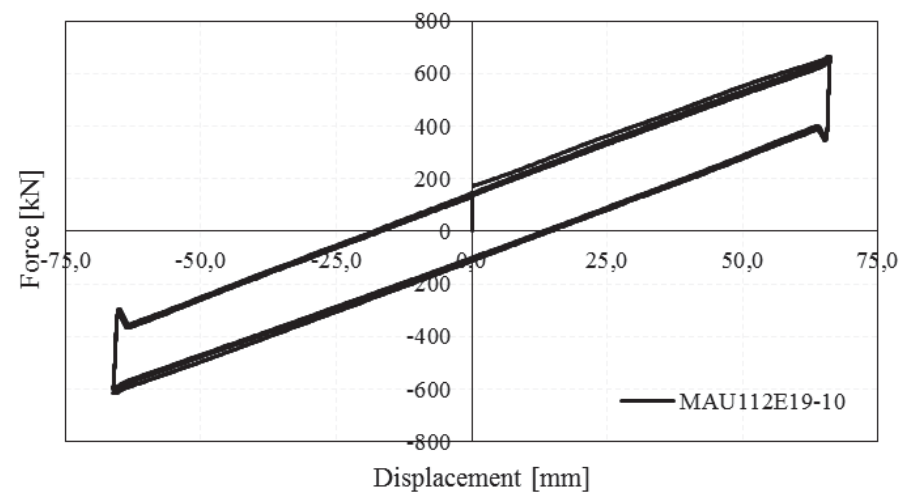

Figure 7: Displacement-force plot for the 3 cycles of the benchmark test.

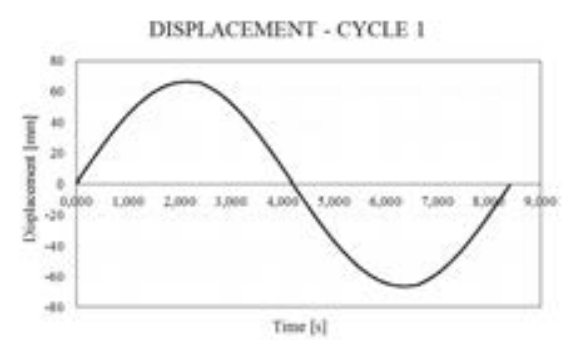

FORCE-CYCLE 1

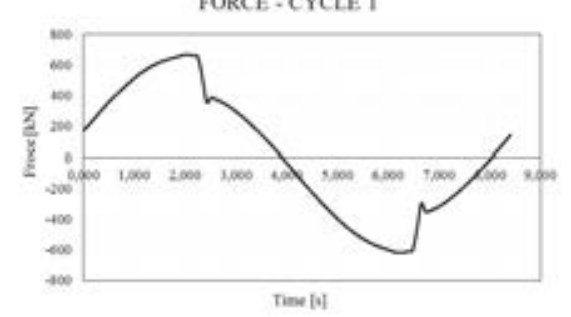

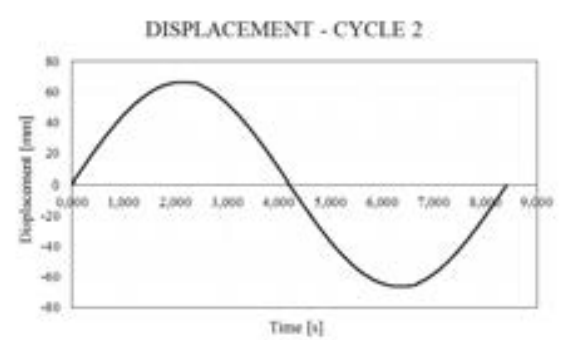

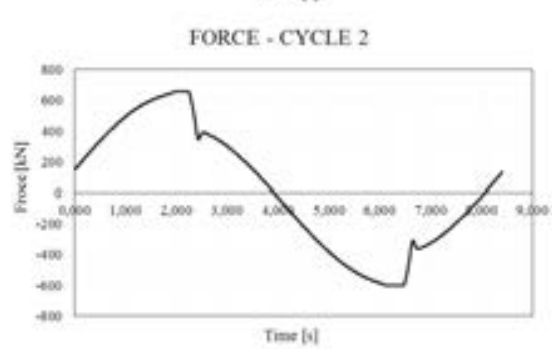

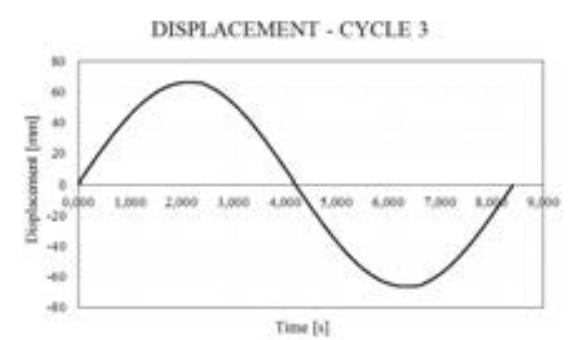

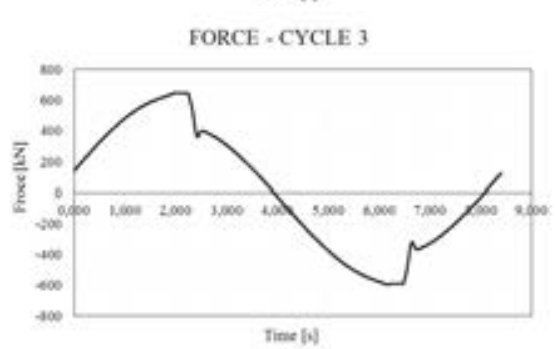

Figure 8: Displacements-time and forces-time plots for the 3 cycles.

Then, the graphical verification of the cycle stability in terms of fluctuation of the test force at each step with respect to the average force is presented in Figures 9-11 for the three cycles. It can be noted a continuous stability of the response has been registered through the 3 cycles investigated. 


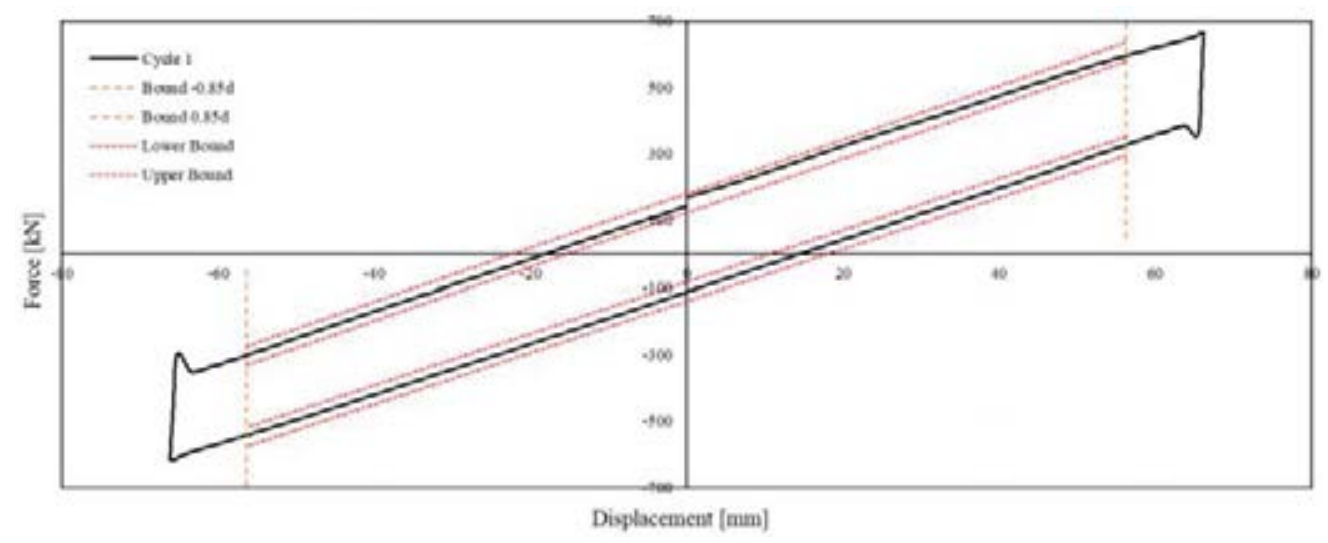

Figure 9: Stability verification for the cycle 1.

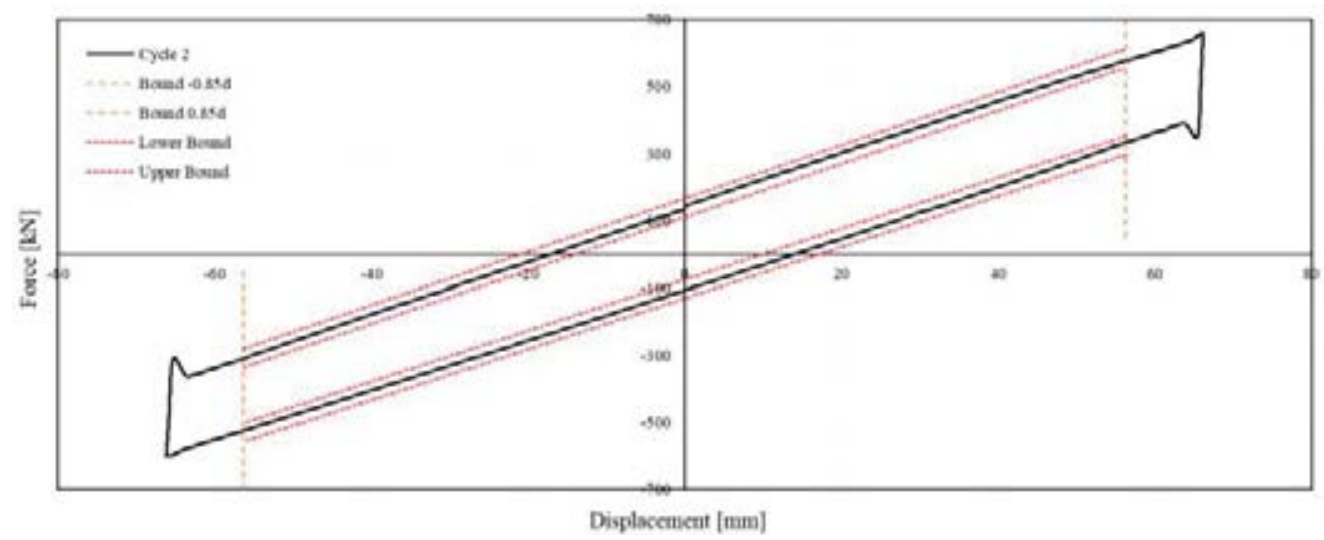

Figure 10: Stability verification for the cycle 2.

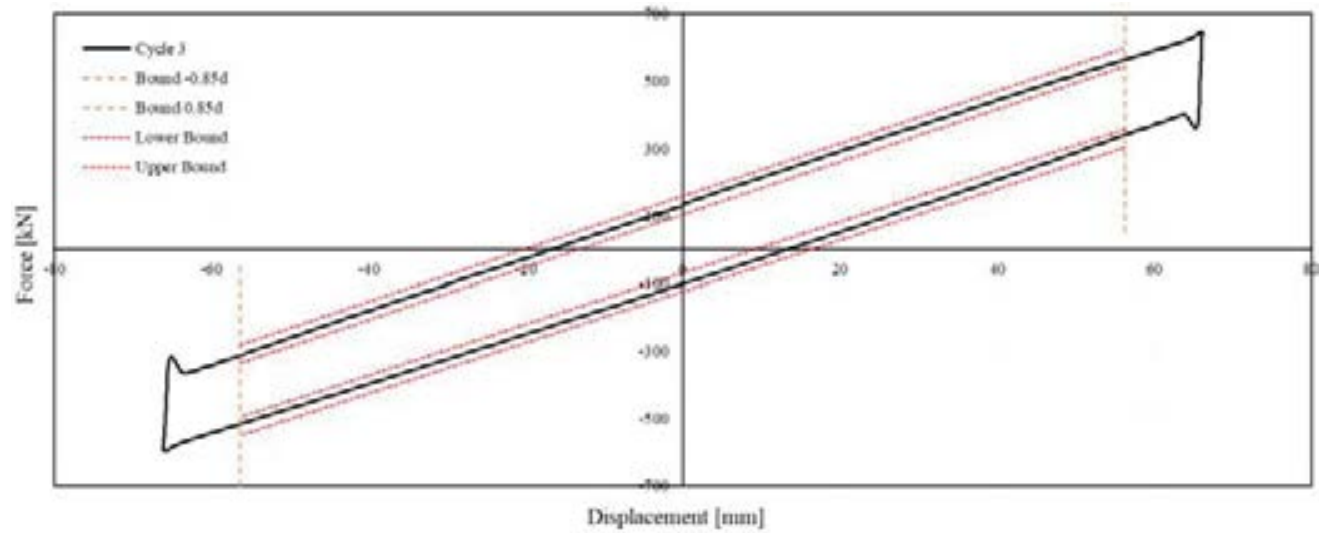

Figure 11: Stability verification for the cycle 3.

In accordance with Equation 3, the value of the dynamic frictional coefficient in the three cycles has been calculated and reported in Table 3. ble 3).

Herein a summary of the dynamic frictional coefficient in the three cycles is reported (Ta-

\begin{tabular}{ccc}
\hline Cycle 1 & Cycle 2 & Cycle 3 \\
\hline $0.5 \%$ & $0.5 \%$ & $0.5 \%$ \\
\hline
\end{tabular}

Table 3: Summary of the dynamic frictional coefficient in the three cycles. 
The UNI EN 15129 [1] states that the average restoring force shall be obtained from the best-fit straight line determined by the least square interpolation of the response $\pm 85 \%$ of the test displacement peak. Fluctuations in horizontal force must be less than $\pm 5 \%$ of the average restoring force, at any level of displacement of the sliding isolator up to $85 \%$ of the test displacement peak. Thus, the procedure to identify for which value of this average restoring force the 5\% interval should be calculated may lead to different interpretations (i.e. with respect to the interval in which the test outcome should be contained in order to satisfy the test). It is clear that this mentioned value should depend on an average of the maximum forces reached during the test in correspondence of the $\pm 85 \%$ of the test displacement peak, but a more straightforward explanation could help the users.

\section{CONCLUSIONS}

The San Giorgio Bridge has been built in a very short time between April 2019 and August 2020 in order to reconnect the highway system of the western part of Italy after the tragedy of the collapse of the Morandi Bridge. The new viaduct has a steel concrete composite closes section for the deck, a structural scheme of continuous beam with a total of 19 spans supported by elliptical concrete box piers with a maximum height of $40 \mathrm{~m}$. The bridge is seismically isolated by means of a composition of friction pendulum devices and multidirectional bearings. One of the most important tasks related to the quality control for the San Giorgio Bridge is related to the qualification and acceptance tests of the seismic devices and bearings planed in the viaduct to isolate the steel-concrete deck from the piers. The testing program for the curved surface sliders was planned also considering the construction and project plan aiming at match the main milestones in terms of deck launching and the time required to perform the test. To do so, the required functional tests have been organized in compliance with the construction plan requirement with the important support and contribution of all the laboratories charged to execute the tests.

A detailed description of the whole testing program with a particular focus on the acceptance tests protocol, as well as a summary of the performance of the devices during the specified static and dynamic tests, has been discussed herein. Despite the demanding testing requirements in the new European norm, the results proved that the proposed design of the isolators successfully fulfilled the performance required for the acceptance of the devices. Differently from other general design projects, in this case the acceptance process corresponds with the certification process, generating many uncommon issues. In fact, in this case, the devices are not commercially produced elements, but have been realized " $a d$ hoc" for the bridge, due to the requirements from the design specifications (e.g., the presence of the 100 meter spans and the presence of only two bearing per each pier have conducted to big dimensions and big forces for the devices). During the implementation of the tests program, many issues came out and have been deeply described through the paper.

Different important suggestions arisen from the experience of the preparation of the seismic acceptance tests.

First of all, it is important that the designer considers, since the design stage, to define devices with testable parameters. The vertical loads are sometimes very high in particular for modern bridges and the testing facilities may have not adequate capacity. The designers should design the structures/infrastructures in way that all the components are testable (potentially in the same geographical region of the construction), based on the current instrumentations availability of the laboratories to avoid significant time and cost increase in the certification/acceptance process. Specific standards may be introduced by technical norms and guidelines in such cases in order to simplify the whole procedure, by keeping the same safety level. A strong cooperation between the testing facilities, the designers, the manufacturers and 
the Construction Supervision team is required in order to define the adequate tests able to assess the performances of the devices and hence guarantee a high level of quality control while at the same time respecting the construction and manufacturing schedule.

\section{AKNOWLEDGMENTS}

The authors would like to acknowledge the manufacturers Maurer SE and FIP-MEC for their professional cooperation during the whole project. A special thanks also goes to the laboratories (Eucentre, SismaLab and the Polytechnic of Milan) for their strong support in the definition of the seismic acceptance tests and for the assistance during the testing campaign. Finally, the authors wish to acknowledge the consortium PerGenova, the designer Italferr, the technical department of RFI and the Commissioning Authorities part of ANAS group for their technical support in the definition and interpretation of the tests.

\section{REFERENCES}

[1] UNI EN 15129 (CEN - Comité Européen de Normalisation, European Code): Antiseismic devices, Brussels; 2018.

[2] D.M. Fenz, M.C. Constantinou, Behaviour of the double concave friction pendulum bearing. Earthquake Engineering and Structural Dynamics, 35(11), 1403-1424, 2006.

[3] V. Quaglini, M. Bocciarelli, E. Gandelli, P. Dubini, Numerical assessment of frictional heating in sliding bearings for seismic isolation. Journal of Earthquake Engineering, 18(8), 1198-1216, 2014.

[4] A. Pavese, M. Furinghetti, C. Casarotti, Experimental assessment of the cyclic response of friction-based isolators under bidirectional motions. Soil Dynamics and Earthquake Engineering, 114, 1-11, 2018.

[5] A. Pavese, M. Furinghetti, C. Casarotti, Investigation of the consequences of mounting laying defects for curved surface slider devices under general seismic input. Journal of Earthquake Engineering, 23(3), 377-403, 2019.

[6] G.M. Calvi, D. Pietra, M. Moratti, Criteri per la progettazione di dispositivi di isolamento a pendolo scorrevole. Progettazione Sismica, 3, 2010.

[7] P.M. Calvi, G.M. Calvi, Historical development of friction-based seismic isolation systems. Soil Dynamics and Earthquake Engineering, 106, 14-30, 2018.

[8] NTC 2018: Supplemento ordinario alla Gazzetta Ufficiale, n. 42 del 20 Febbraio 2018. Ministero delle Infrastrutture e dei Trasporti, Ministerial Decree DM 17 Gennaio 2018 "Aggiornamento delle Norme tecniche per le costruzioni" (in italian).

[9] UNI EN 1337 part 2 (CEN - Comité Européen de Normalisation, European Code): Structural bearings - Part 2: Sliding elements, Brussels; 2004. 